\title{
Factores personales y ambientales asociados con el desplazamiento activo al colegio de los escolares españoles
}

\author{
Villa González, Emilio \\ Tutora: Dr. Palma Chillón Garzón \\ Dpto. Educación Física y Deportiva \\ Facultad de C.C de la A.F y D (Universidad de Granada) \\ Granada, Diciembre, 2011
}




\section{ÍNDICE}

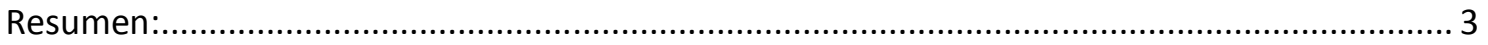

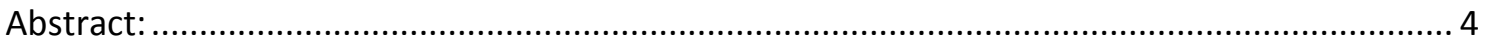

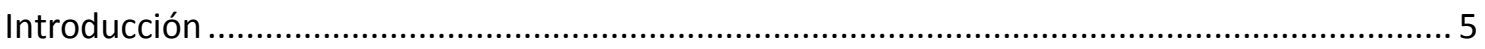

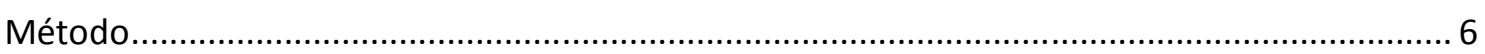

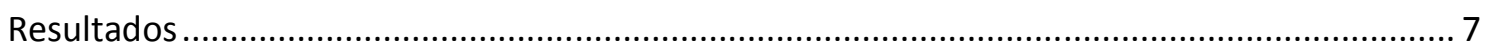

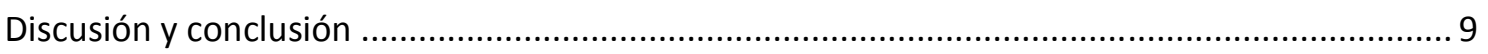

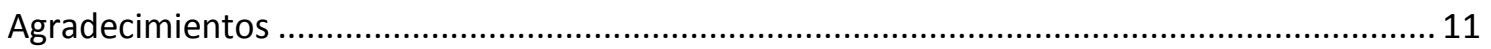

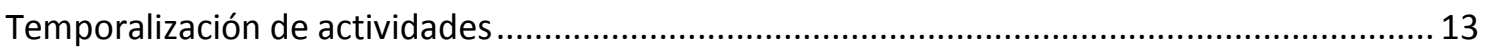

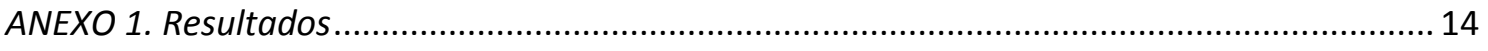

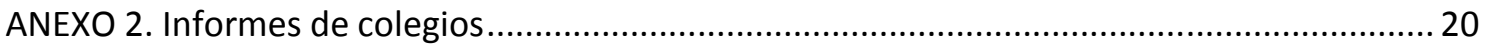




\section{Resumen:}

Objetivos: Los factores personales y ambientales pueden influir en el transporte activo al colegio. Los objetivos fueron: a) examinar los patrones de los desplazamientos a la escuela entre los escolares $\mathrm{y}, \mathrm{b}$ ) analizar la asociación de factores personales y ambientales con el desplazamiento activo a la escuela.

Método: Los participantes fueron 745 escolares de 8,9 años (SD: 1,7), (363 niñas), de cuatro colegios en la provincia de Granada. Los escolares rellenaron un cuestionario sobre el modo de desplazamiento, factores personales y ambientales. Las diferencias en el modo de desplazamiento al colegio en función de género y edad se analizaron con Chi-cuadrado. La relación de los factores personales y ambientales con el desplazamiento activo al colegio se analizó con regresión logística binaria ajustada por género y edad.

Resultados: Un total de 57,2\% de los escolares realizó el trayecto caminando, el 40,3\% utilizó coche, un 1,2\% moto, el $0,7 \%$ utilizó el autobús y la bicicleta un $0,6 \%$. Hubo diferencias significativas según la edad para el desplazamiento andando y en coche $(\mathrm{P}=$ 0,003). Los niños ${ }^{1}$ que viajaban solos eran más activos que los que viajaban acompañados $(10.049,3.950$ - 25.567\%). Los niños que vivían cerca del colegio eran más activos que los que vivían más lejos (16.439, 5,717-47,271\%). Los niños que percibían caminos seguros eran más activos que aquellos que percibían caminos inseguros $(2.365,1,665-3,360 \%)$.

Conclusión: Intervenciones a nivel familiar y estrategias ambientales en los caminos al colegio, podrían ser medidas eficaces para aumentar el desplazamiento activo al colegio.

Palabras clave: Actividad física, Desplazamiento activo al colegio, estudiantes, factores personales, factores ambientales.

\footnotetext{
${ }^{1}$ Cuando utilicemos el nombre en masculino de niños o hijos, nos referimos igualmente a niñas o hijas
} 


\begin{abstract}
:
Objectives: Personal and environmental factors might influence active commuting to school. We aimed to: a) examine the patterns of commuting to school in schoolchildren and, b) to analyze the association of personal and environmental factors with active commuting to school.
\end{abstract}

Methods: Participants were 745 schoolchildren aged 8, 9 years (SD: 1,7) (363 girls), from four schools in the province of Granada. A questionnaire about mode of commuting, personal and environmental factors was self-reported by children. Differences in mode of commuting to school (active vs. passive) according to gender and age were examined using chi-square. Associations between personal and environmental factors with active commuting to school were examined using binary logistic regression adjusting for gender and age.

Results: A total of $57.2 \%$ children walked to school, $40.3 \%$ used the car, $1.2 \%$ motorcycled, $0.7 \%$ used the bus and $0.6 \%$ bicycled. There were age differences for walking and car $(\mathrm{P}=0.003)$. Children who traveled alone were more likely to be active than those who traveled with somebody else (10.049, 3,950- 25,567\%). Children living close to school were more active than those living far (16.439, 5,717- 47,271\%). Children who perceived a safer way were more active than those perceiving a non-safe way to school $(2.365,1,665-3,360 \%)$.

Conclusion: Family-based interventions and environmental strategies in the way to school could be effective initiatives to increase the rate of active commuting to school.

Key Words: Physical- Activity; Active commuting to school; Personal factors; Environmental factors. 


\section{Introducción}

El sedentarismo se asocia con la obesidad y los factores de riesgo cardiovasculares. Este sedentarismo, parece tener origen en la infancia y la adolescencia ${ }^{1}$. Los esfuerzos para desarrollar un estilo de vida activo en los niños, parece ser una estrategia exitosa para aumentar la actividad física, disminuyendo así los índices de obesidad. Se ha recomendado, que los niños acumulen 2.1 horas de actividad física cada día para recibir los beneficios de salud ${ }^{2}$. Un ejemplo, de estilo de vida activa es caminar o ir en bicicleta a la escuela, en vez de ir en coche. El desplazamiento activo al colegio podría contribuir a combatir la epidemia de obesidad internacional que se extiende durante la infancia, y que puede establecerse como un hábito de actividad física que perdure en la adolescencia y la edad adulta ${ }^{3}$. Las tasas de transporte activo, han disminuido en los últimos 30 años drásticamente. En 1995, sólo el 28\% de los niños de entre 5-15 años en EE.UU, viviendo a $1,6 \mathrm{~km}$ de la escuela, realizaba el trayecto caminando (EEUU. Departamento de transporte de 1995). Mayores porcentajes de desplazamiento activo existen actualmente en población española ${ }^{4,5}$.

Existe ligera evidencia sobre los patrones de desplazamiento activo de los escolares españoles en población adolescente ${ }^{4,5}$. A partir del estudio AVENA en el que participaron 2183 adolescentes con edades entre 13, y 18,5 años, se obtuvo que $64.8 \%$ de los adolescentes realizaban el desplazamiento al colegio de forma activa ${ }^{4}$ En el estudio AFINOS que incluye 2029 adolescentes de 13 a 17 años de Madrid y provincia, un $56.8 \%$ se desplazaron de forma activa al colegio ${ }^{5}$. Sin embargo, en la actualidad existe menos evidencia de cómo son los patrones de desplazamiento de los niños españoles hacia el colegio.

Son diversos los estudios que se han interesado en analizar qué factores determinan el desplazamiento activo de los jóvenes el colegio ${ }^{6,}$ 7. Los factores sociales y las características del medio ambiente son predictores importantes del desplazamiento activo al colegio ${ }^{6}$. Existe evidencia de que las actitudes previas, el apoyo social y las percepciones del medio ambiente están asociadas al desplazamiento activo al colegio de los niños, siendo estas asociaciones moderadas por la influencia de la distancia al colegio $^{8}$. En niños de Suiza, se ha estudiado la relación entre el modo de desplazamiento y factores personales y ambientales, encontrando correlaciones significativas ${ }^{9}$. En 235 
niños de 5 a 6 años y 677 niños de 10 a 12 años de 19 colegios de primaria de Melbourne, Australia, se estudió la relación entre factores personales, familiares, sociales y ambientales, con el desplazamiento activo al colegio ${ }^{10}$. Debido a la especificidad que los factores personales y ambientales tienen en cada contexto geográfico y cultural, es de interés analizar estos factores en España.

Por tanto los objetivos del presente estudio son: a) conocer los patrones de desplazamiento al colegio de los niños participantes de la provincia de Granada, España y b) estudiar qué factores personales y ambientales están asociados al desplazamiento activo al colegio.

\section{Método}

Los datos obtenidos en el presente estudio, fueron recogidos de una muestra formada por 745 escolares (edad de 6-12 años; media=8,9 y desviación típica=1,7) de Educación Primaria (382 niños y 363 niñas), pertenecientes a cuatro colegios de la provincia de Granada de las localidades de Salobreña $(\mathrm{N}=221)$, Huétor Vega con dos centros escolares $(\mathrm{N}=165$ y $\mathrm{N}=208)$ y Santa Fé $(\mathrm{N}=151)$.

Los colegios participantes pertenecen a municipios integrados en una iniciativa del área de Medio Ambiente de la Diputación de Granada cuyo objetivo es favorecer caminos seguros y saludables al colegio en los escolares. Los directores de los centros educativos recibieron una hoja informativa sobre la naturaleza y propósito del estudio. Todos los directores/as, firmaron el documento y aceptaron colaborar en el proyecto, además, las familias firmaron un consentimiento informado, autorizando así, el uso y participación del hijo/a en el estudio.

Los responsables del estudio implementaron inicialmente un cuestionario a cada niño aplicándose por grupos de clase. Los niños completaron los cuestionarios en los centros educativos ayudados por su tutor y, en algunos casos, con la ayuda de los investigadores del presente estudio. El modo de desplazamiento al colegio se obtuvo con la pregunta: “CCómo vas al cole? A pie, en coche, en bici o en moto”. Dicha respuesta se categorizó en activos (si se desplazan andando o en bicicleta) y pasivos (si se desplazan en coche, moto o autobús). Además, se preguntaba sobre factores personales (barreras que impiden andar al colegio, grado de diversión en el camino al 
colegio, con quién van acompañados al colegio) y ambientales (tiempo del trayecto al colegio, distancia al colegio y grado de seguridad del trayecto). Las barreras percibidas de andar al colegio fueron: lejanía, tiempo, tráfico, falta de aceras, miedo, familias lo impiden, cansancio y meteorología). Partiendo de ésta, se creó la variable de barrera global identificando el número total de barreras percibidas.

El criterio de inclusión fue aquellos que tenían datos completos en el modo de desplazamiento, resultando en un total de 672 niños. El análisis estadístico se basó en datos descriptivos en el modo de desplazamiento para la muestra total y atendiendo al género (niños y niñas) y a grupos de edad (6-9 años y 10-12 años). A través del test chicuadrado se estudiaron las diferencias en los modos de desplazamiento al colegio de andar y en coche atendiendo al género y a la edad. No se estudiaron en el resto de modos de desplazamiento por no existir suficiente muestra. Las asociaciones entre los factores personales y ambientales y el desplazamiento activo al colegio se estudiaron utilizando la regresión binaria logística basada en Odds Ratios e intervalos de confianza (95\%). En dicho análisis, la variable dependiente fue el modo de desplazamiento (activo vs pasivo), las variables independientes fueron los factores personales y ambientales que se analizaron individualmente; los análisis se ajustaron por género y edad. Previamente se estudiaron las interacciones de dichas asociaciones con género y edad y al no ser significativas, se analizó la muestra completa. Las referencias de los factores personales y ambientales fueron las categorías, a priori, menos propicias para el desplazamiento activo al colegio. El programa utilizado para el análisis estadístico fue el SPSS.18 y el nivel de significación se fijó en 0,05 .

\section{Resultados}

Los patrones del desplazamiento al colegio del alumnado se muestran en las figuras 1,2 y 3 . Un $57,5 \%$ de los escolares encuestados viajaron al colegio de forma activa y $42,5 \%$ se desplazaron de forma pasiva. De los activos, un $56,8 \%$ lo hicieron andando y un $0,7 \%$ lo realizó en bicicleta. El transporte pasivo más utilizado fue el coche $(40 \%)$. Atendiendo al género, no existieron diferencias significativas en el modo de transporte hacia el colegio de andar y coche $(\mathrm{p} \geq 0.603)$ (Figura 2). Atendiendo a los grupos de edad, sí existieron diferencias significativas para los modos de andar y coche 
$(\mathrm{p}=0,003)$ (Figura 3), siendo el grupo de 10-12 años más activo que el grupo de 6-9 años.

Los datos descriptivos de los factores personales y ambientales se muestran en la tabla 1. Las barreras que más percibieron los escolares como limitantes para desplazarse al colegio de forma activa fueron la lejanía (37,9\%), el tiempo (35,3\%), y el impedimento de las familias $(32,0 \%)$. En cuanto a la forma de acompañamiento, un $78,4 \%$ de los escolares van a los colegios acompañados de su padre/madre, el $8,1 \%$ van con amigos/as, el 6,9\% con abuelos, el 10,1\% van solos, un 7,4\% van con vecinos/as, un $0,3 \%$ van con hermanos/as y un $0,2 \%$ con cuidador/a. Un $43,5 \%$ de los escolares viven a menos de 300 metros del colegio y el $82 \%$ de los escolares tardaba menos de 10 minutos en desplazarse al colegio.

Las asociaciones entre los factores personales y ambientales con el desplazamiento activo al colegio se expresan en la tabla 2. Los escolares que percibieron menos número de barreras (de 1 a 3), tienen una probabilidad mayor de ser activos que los que encontraron un mayor número de barreras (de 4 a 7), (OR; 6,845, CI; 0,910-51,503), si bien el resultado no es significativo $(p=0,066)$. Los escolares que se desplazaban solos al colegio tenían más probabilidad de ser activos que los que lo hacían con algún acompañante $(10,049 ; 3,950-25,567)$, y los que se desplazaban con los padres/madres tenían menos probabilidad de ser activos que los que no lo hacían $(0,213 ; 0,132-0,344)$. Respecto a los factores ambientales, se obtuvieron diferencias significativas para la distancia al colegio y la seguridad en el camino. Los que viven a una distancia de 1 a $300 \mathrm{~m}$ del colegio fueron más activos que los que viven a más de $2 \mathrm{~km}(16,439 ; 5,717$ 47,271). Los adolescentes que perciben más seguridad fueron más activos que los que perciben más inseguridad en el camino al colegio $(2,365 ; 1,665-3,360)$. 


\section{Discusión y conclusión}

Los resultados obtenidos en el presente estudio sugieren que más de la mitad de los escolares son activos en el desplazamiento al colegio. Los niños que se desplazaban solos al colegio, vivían cerca del colegio y percibían más seguridad en el camino, eran más propensos a ser activos que sus contrarios.

Alrededor del 57\% de los niños escolares realizaron un desplazamiento activo al colegio. Datos ligeramente superiores se obtuvieron en adolescentes españoles $(<65 \%)^{4}$ y similares en niños de Bélgica $(<60 \%)^{7}$. En Inglaterra, sólo un $48 \%$ de niños iban al colegio de forma activa ${ }^{8}$. Sin embargo, los niños de Suiza muestran mayores porcentajes de desplazamiento activo, siendo el $78 \%$ de ellos los que van al colegio andando o en bicicleta 9 .

En cuanto a la relación del transporte activo en niños y niñas no se encontraron en el actual estudio resultados significativos. Sí se encontró evidencia, en la forma de desplazarse y la edad, aumentando el transporte activo con el aumento de la edad. En otros estudios existentes no se encontraron diferencias significativas entre niños ${ }^{7,8}$.

Los factores personales que más se asociaron a un desplazamiento activo fueron: una menor percepción de las barreras de lejanía, tiempo e impedimento de la familia y el ir solos al colegio. Por el contrario, el ir con padre/madre se asociaba a un desplazamiento pasivo, indicativo de que si van con los padres es porque los llevan en coche. En la edad infantil la influencia de las familias se hace más patente analizando en algunos estudios la influencia negativa de las familias en los desplazamiento activos al colegio $^{11}$. Otros resultados concluyen que las actitudes de los padres, las preocupaciones por la seguridad, la presencia de apoyo social de los padres y los amigos y la posibilidad de hacer el trayecto acompañado, pueden ser predictores de desplazamiento activo 9 . Los niños que recibían apoyo de los compañeros y la familia y vivían en entornos con gran apoyo social, tenían más probabilidad de ser activos en el camino al colegio ${ }^{9}$. En España se ha estudiado la asociación de algunos factores personales y su influencia en el transporte activo al colegio en adolescentes del estudio AVENA y se demostró que el desplazamiento activo al colegio y su duración pueden influir positivamente en el rendimiento cognitivo en las adolescentes ${ }^{5}$. 
Respecto a los factores ambientales, se encontró que la distancia de casa al colegio y la seguridad del camino son los factores más relacionados con el desplazamiento activo. La distancia es una barrera importante en la realización de transporte activo y el primer factor de predicción en el del modo de desplazamiento activo entre jóvenes ${ }^{8,12}$. Se estudió el efecto moderador de la distancia, y se observó que las actitudes, en el desplazamiento activo son más importantes en distancias cortas y ante problemas de seguridad en el camino ${ }^{8}$. Aunque no existe un criterio universal, algunos autores comentan que una distancia de 2.5 millas se considera como alcanzables por caminantes y ciclistas en jóvenes ${ }^{13}$. La seguridad en el camino ha sido también mencionada en otros estudios como factor determinante para el desplazamiento activo al colegio ${ }^{9}$. La mayoría de los estudios analizando factores ambientales se han realizado en EEUU y Australia y se requerirían más investigaciones en Europa y Asia, porque el contexto geográfico y cultural difiere. Así lo muestran otros estudios que relacionan los factores ambientales como el clima estacional, con el desplazamiento activo al colegio ${ }^{14}$. Además, es de interés incorporar más variables de tipo ambiental, como el estatus social de las familias y su relación con el ambiente ${ }^{6}$.

Una limitación es el uso del cuestionario, el cuál fue creado específicamente para dicho estudio sin haber sido constatado su validez y fiabilidad. Se necesitan estudios futuros, que aporten instrumentos de desplazamiento activo a los colegios válidos y fiables y en lengua castellana. Cómo punto fuerte, destacar que éste estudio es, bajo nuestro conocimiento, el primero que se realiza de desplazamiento al colegio con niños españoles.

La consistencia de la mayoría de los resultados, confirma la importancia de mejorar el diseño urbano para aumentar el bienestar y la seguridad en los desplazamientos activos, y a su vez, concienciar a las familias de estos escolares favorecer dicho comportamiento. Se hace patente la creación de políticas educativas destinadas a ayudar al transporte activo hacia el colegio. 


\section{Agradecimientos}

Queremos agradecer la colaboración de todos los componentes del grupo de investigación, que hicieron posible este estudio, además de los colaboradores de la Diputación de Granada. También reconocemos la ayuda a todos los niños que participaron en el estudio, como a sus familias y maestros que colaboraron en el mismo. 


\section{Referencias}

1.Freedman DS, Shear CL, Burke GL, Srinivasan SR, Webber LS, Harsha DW, et al. Persistence of juvenile-onset obesity over eight years: the Bogalusa Heart Study. Am J Public Health. 1987;77(5):588-92.

2.Corbin CB, Pangrazi RP. Are American children and youth fit? Res Q Exerc Sport. 1992;63(2):96-106.

3.Tudor-Locke C, Ainsworth BE, Popkin BM. Active commuting to school - An overlooked source of childrens' physical activity? Sports Med. 2001;31(5):309-13.

4.Chillon P, Ortega FB, Ruiz JR, Perez IJ, Martin-Matillas M, Valtuena J, et al. Socio-economic factors and active commuting to school in urban Spanish adolescents: the AVENA study. Eur J Public Health. 2009;19(5):470-6.

5.Martinez-Gomez D, Ruiz JR, Gomez-Martinez S, Chillon P, Rey-Lopez JP, Diaz LE, et al. Active Commuting to School and Cognitive Performance in Adolescents The AVENA Study. Arch Pediat Adol Med. 2011;165(4):300-5.

6.Hume C, Jorna M, Arundell L, Saunders J, Crawford D, Salmon J. Are children's perceptions of neighbourhood social environments associated with their walking and physical activity? J Sci Med Sport. 2009;12(6):637-41.

7.D'Haese S, De Meester F, De Bourdeaudhuij I, Deforche B, Cardon G. Criterion distances and environmental correlates of active commuting to school in children. Int J Behav Nutr Phy. $2011 ; 8$.

8.Panter JR, Jones AP, van Sluijs EMF, Griffin SJ. Attitudes, social support and environmental perceptions as predictors of active commuting behaviour in school children. J Epidemiol Commun H. 2010;64(1):41-8.

9.Bringolf-Isler B, Grize L, Mader U, Ruch N, Sennhauser FH, Braun-Fahrlander C, et al. Personal and environmental factors associated with active commuting to school in Switzerland. Prev Med. 2008;46(1):67-73.

10.Timperio A, Ball K, Salmon J, Roberts R, Giles-Corti B, Simmons D, et al. Personal, family, social, and environmental correlates of active commuting to school. Am J Prev Med. 2006;30(1):45-51.

11.Lee MC, Orenstein MR, Richardson MJ. Systematic Review of Active Commuting to School and Children's Physical Activity and Weight. J Phys Act Health. 2008;5(6):930-49.

12.Davison K, Werder, JL, Lawson, CT. Children's active commuting to school: current knowledge and future directions. Prev Chronic Dis. 2008;5(A):100.

13.Nelson NM, Foley E, O'Gorman DJ, Moyna NM, Woods CB. Active commuting to school: How far is too far? Int J Behav Nutr Phy. 2008;5:-.

14.Borrestad LAB, Andersen LB, Bere E. Seasonal and socio-demographic determinants of school commuting. Prev Med. 2011;52(2):133-5. 


\section{Temporalización de actividades}

A continuación pasaré a describir la temporalización de las actividades que hemos realizado durante el tiempo de elaboración de dicho estudio.

En la primera fase, contactamos con la Diputación de Granada, para mostrarles, nuestro interés en participar, en el estudio que tenían planteado, para el año 2011, de Itinerarios saludables y seguros". Nuestra aportación iría encaminada al aporte de carácter científico, dentro del estudio que se iba a realizar.

Cuando nos incorporamos al proyecto (enero 2011), realizamos diferentes reuniones de planificación de las actividades que a continuación se detallan (febrero 2011). En este mes, procedimos a la entrega de de los cuestionarios en los centros y realizamos las respectivas visitas a los colegios. Durante los meses siguientes, se realizaron algunas actividades, de formación continua, que estaban contempladas dentro de los proyectos de centro, porque previamente al inicio del curso la Diputación de Granada, remitió dichas actividades a los centros, para la colaboración en el proyecto. Estas iban encaminadas educar en valores, como la educación vial, y tuvimos la suerte, de poder colaborar en ellas.

Entre los meses de mayo y junio de 2011, se elaboró un informe, para cada uno de los colegios. Un ejemplo de dicho informe se añade en el (Anexo 3).

Por último en el mes de noviembre, se redactó un Abstract, que fue presentado, en formato de Póster, en el "International Congress of Physical Activity in the Prevention and Treatmen of Chronic Deseases", celebrado en Granada. El abstract, el póster y el certificado correspondiente a la presentación en el congreso se encuentran en (Anexo4). 
Tabla 1. Factores personales y ambientales según el modo de desplazamiento

\begin{tabular}{|c|c|c|c|}
\hline & Activos, n (\%) & Pasivos, n (\%) & Todos, n (\%) \\
\hline \multicolumn{4}{|l|}{ Factores personales } \\
\hline \multicolumn{4}{|l|}{ Barreras de ir a pie } \\
\hline Lejanía & $12(19,4)$ & $115(42,1)$ & $127(37,9)$ \\
\hline Tiempo & $12(19,4)$ & $106(39,0)$ & $118(35,3)$ \\
\hline Tráfico & $6(9,7)$ & $40(14,7)$ & $46(13,8)$ \\
\hline Falta de aceras & $5(8,1)$ & $33(12,1)$ & $38(11,4)$ \\
\hline Miedo & $8(12,9)$ & $48(17,6)$ & $56(16,8)$ \\
\hline Familias impiden & $13(21,0)$ & $94(34,6)$ & $107(32,0)$ \\
\hline Cansancio & $11(17,7)$ & $52(19,1)$ & $63(18,9)$ \\
\hline Meteorología & $15(24,2)$ & $41(15,1)$ & $56(16,8)$ \\
\hline \multicolumn{4}{|l|}{ Barrera global } \\
\hline 1-3 barreras & $60(98,4)$ & $244(90,0)$ & $304(91,6)$ \\
\hline 4-7 barreras & $1(1,6)$ & $27(2,0)$ & $28(8,4)$ \\
\hline \multicolumn{4}{|l|}{ Diversión } \\
\hline Muy bien & $205(53,2)$ & $123(44,1)$ & $328(49,4)$ \\
\hline Ni bien ni mal & $167(43,4)$ & $147(52,7)$ & $314(47,3)$ \\
\hline Mal & $13(3,4)$ & $9(3,2)$ & $22(3,3)$ \\
\hline \multicolumn{4}{|l|}{ Acompañamiento } \\
\hline Padre/madre & $262(68,8)$ & $258(95,5)$ & $520(78,4)$ \\
\hline Amigo & $38(9,9)$ & $16(5,7)$ & $54(8,1)$ \\
\hline Abuelos & $31(8,1)$ & $15(5,3)$ & $46(6,9)$ \\
\hline Vecinos & $28(7,3)$ & $21(7,4)$ & $49(7,4)$ \\
\hline Sólo & $62(16,2)$ & $5(1,8)$ & $67(10,1)$ \\
\hline Hermanos & $1(0,3)$ & $1(0,4)$ & $2(0,3)$ \\
\hline Cuidador & $1(0,3)$ & $0(0)$ & $1(0,2)$ \\
\hline \multicolumn{4}{|c|}{ Factores ambientales } \\
\hline \multicolumn{4}{|l|}{ Distancia al colegio } \\
\hline $1-300 \mathrm{~m}$ & $134(57,0)$ & $34(22,5)$ & $168(43,5)$ \\
\hline $301-500 \mathrm{~m}$ & $49(20,9)$ & $34(22,5)$ & $83(21,5)$ \\
\hline $501-1000 \mathrm{~m}$ & $41(17,4)$ & $45(29,8)$ & $86(22,3)$ \\
\hline $1001-2000 \mathrm{~m}$ & $6(2,6)$ & $18(11,9)$ & $24(6,2)$ \\
\hline$>2000$ & $5(2,1)$ & $20(13,2)$ & $25(6,5)$ \\
\hline \multicolumn{4}{|l|}{ Tiempo al colegio } \\
\hline $1-10 \mathrm{~min}$ & $311(84,5)$ & $205(78,5)$ & $516(82,0)$ \\
\hline $11-30 \mathrm{~min}$ & $49(13,3)$ & $49(18,8)$ & $98(15,6)$ \\
\hline $31-44 \mathrm{~min}$ & $3(0,8)$ & $3(1,1)$ & $6(1,0)$ \\
\hline $45-60 \mathrm{~min}$ & $5(1,4$ & $4(1,5)$ & $9(1,4)$ \\
\hline \multicolumn{4}{|l|}{ Seguridad } \\
\hline Si es seguro & $190(50,4)$ & $83(29,4)$ & $273(41,4)$ \\
\hline No es seguro & $187(49,3)$ & $199(70,6)$ & $386(58,6)$ \\
\hline
\end{tabular}


Tabla 2. Odds ratios del desplazamiento activo al colegio para factores personales y ambientales

Desplazamiento al colegio (Activo vs Pasivo)

\begin{tabular}{|c|c|c|c|c|}
\hline & $\mathrm{N}$ & OR & $95 \% \mathrm{CI}$ & $\mathrm{P}$ \\
\hline \multicolumn{5}{|c|}{ Factores personales } \\
\hline \multicolumn{5}{|c|}{ Barreras de ir a pie $^{\text {a }}$} \\
\hline \multirow[t]{2}{*}{ Lejanía } & 138 & 1 & Reference & \\
\hline & 257 & 3,110 & $1,579-6,123$ & 0,001 \\
\hline \multirow{2}{*}{ Tiempo } & 128 & 1 & Reference & \\
\hline & 266 & 2,655 & $1,350-5,221$ & 0,005 \\
\hline \multirow[t]{2}{*}{ Tráfico } & 54 & 1 & Reference & \\
\hline & 340 & 1,602 & $0,647-3,970$ & 0,309 \\
\hline \multirow[t]{2}{*}{ Falta de aceras } & 42 & 1 & Reference & \\
\hline & 352 & 1,637 & $0,609-4,400$ & 0,329 \\
\hline \multirow[t]{2}{*}{ Miedo } & 62 & 1 & Reference & \\
\hline & 332 & 1,435 & $0,636-3,238$ & 0,384 \\
\hline \multirow[t]{2}{*}{ Familias impiden } & 121 & 1 & Reference & \\
\hline & 327 & 1,978 & $1,019-3,842$ & 0,044 \\
\hline \multirow[t]{2}{*}{ Cansancio } & 75 & 1 & Reference & \\
\hline & 319 & 1,083 & $0,527-2,227$ & 0,828 \\
\hline \multirow[t]{2}{*}{ Meteorología } & 76 & 1 & Reference & \\
\hline & 318 & 0,571 & $0,291-1,122$ & 0.595 \\
\hline \multicolumn{5}{|l|}{ Barrera global } \\
\hline 4-7 barreras & 28 & 1 & Reference & \\
\hline 1-3 barreras & 304 & 6,845 & $0,910-51,503$ & 0,062 \\
\hline \multicolumn{5}{|l|}{ Diversión } \\
\hline Mal & 22 & 1 & Reference & \\
\hline Ni bien ni mal & 314 & 0,747 & $0,308-1,814$ & 0,519 \\
\hline Muy bien & 328 & 1,261 & $0,518-3,067$ & 0,609 \\
\hline \multicolumn{5}{|l|}{ Acompañamiento $^{b}$} \\
\hline \multirow[t]{2}{*}{ Padre/madre } & 153 & 1 & Reference & \\
\hline & 582 & 0,213 & $0,132-0,344$ & $<0,001$ \\
\hline \multirow[t]{2}{*}{ Amigo } & 674 & 1 & Reference & \\
\hline & 62 & 1,627 & $0,880-3,011$ & 0,121 \\
\hline \multirow[t]{2}{*}{ Abuelos } & 680 & 1 & Reference & \\
\hline & 56 & 1,856 & $0,970-3,552$ & 0,062 \\
\hline \multirow[t]{2}{*}{ Vecinos } & 677 & & Reference & \\
\hline & 59 & 0,926 & $0,512-1,676$ & 0,801 \\
\hline \multirow{2}{*}{ Sólo } & 662 & 1 & Reference & \\
\hline & 74 & 10,049 & $3,950-25,567$ & $<\mathbf{0 , 0 0 1}$ \\
\hline
\end{tabular}

Factores ambientales

\section{Distancia al colegio}

$>2000$

1001-2000 m

$501-1000 \mathrm{~m}$

$301-500 \mathrm{~m}$

$\begin{array}{ll}25 & 1 \\ 24 & 0,678 \\ 86 & 3,657 \\ 83 & 6,068\end{array}$

Reference

$0,344-5,155$

0,678

$1,252-10,683$

$2,056-17,850$

0,001

Máster Universitario en Investigación de Actividad Física y Deporte 
$1-300 \mathrm{~m} \quad 168 \quad 16,439 \quad 5,717-47,271 \quad<\mathbf{0 , 0 0 1}$

Tiempo al colegio

$\begin{array}{lllll}45-60 \mathrm{~min} & 9 & 1 & \text { Reference } & \\ 31-44 \mathrm{~min} & 6 & 0,775 & 0,096-6,272 & 0,811 \\ 11-30 \mathrm{~min} & 98 & 0,808 & 0,200-3,256 & 0,764 \\ 1-10 \mathrm{~min} & 516 & 1,223 & 0,319-4,694 & 0,769\end{array}$

Seguridad

$\begin{array}{llll}\text { No es seguro } & 386 & 1 & \text { Reference }\end{array}$

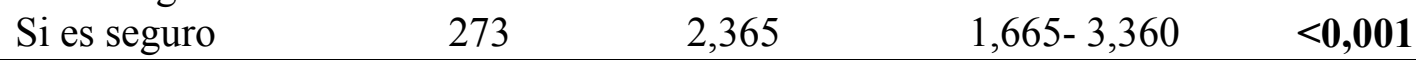

M, metro s; min, minutos. ${ }^{a}$ la referencia es manifestar la barrera; ${ }^{b}$ la referencia es no ser acompañado 


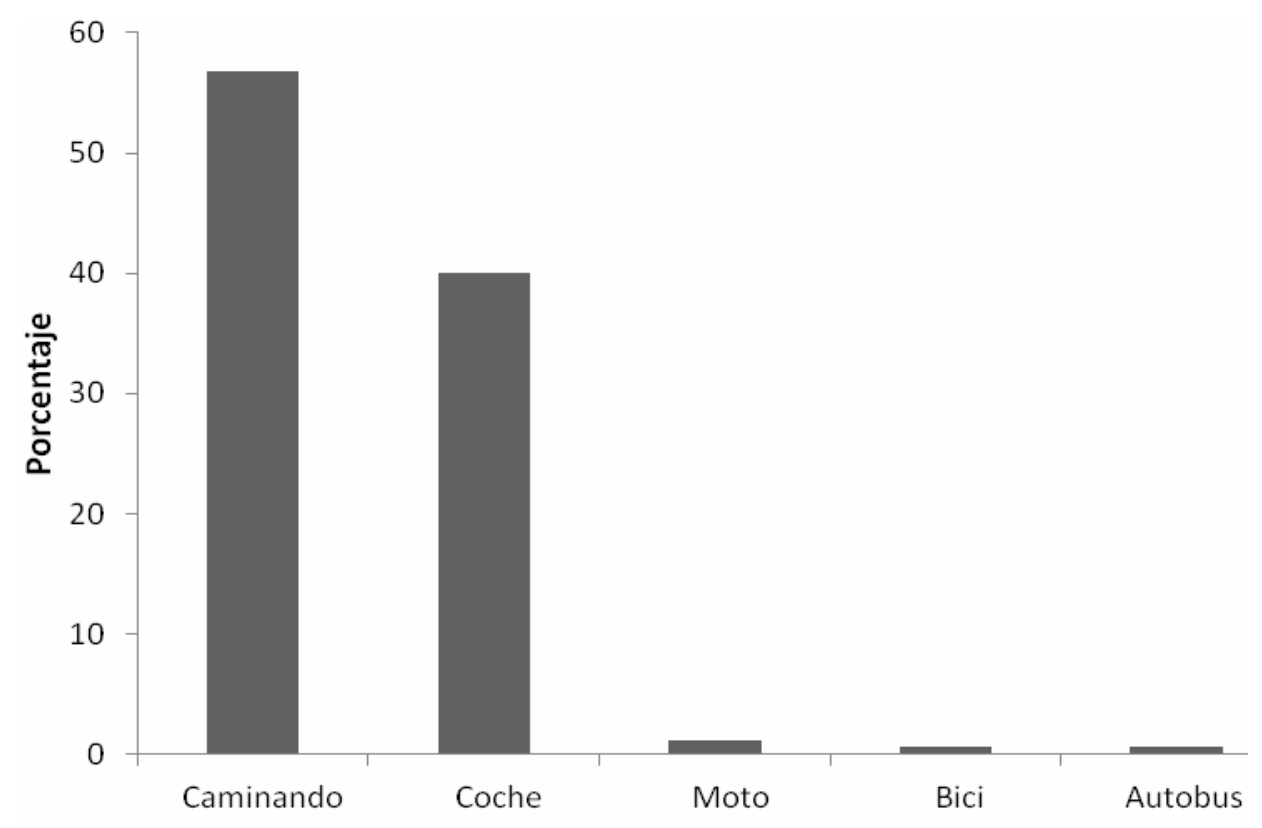

Figura 1. Modo de desplazamiento al colegio 


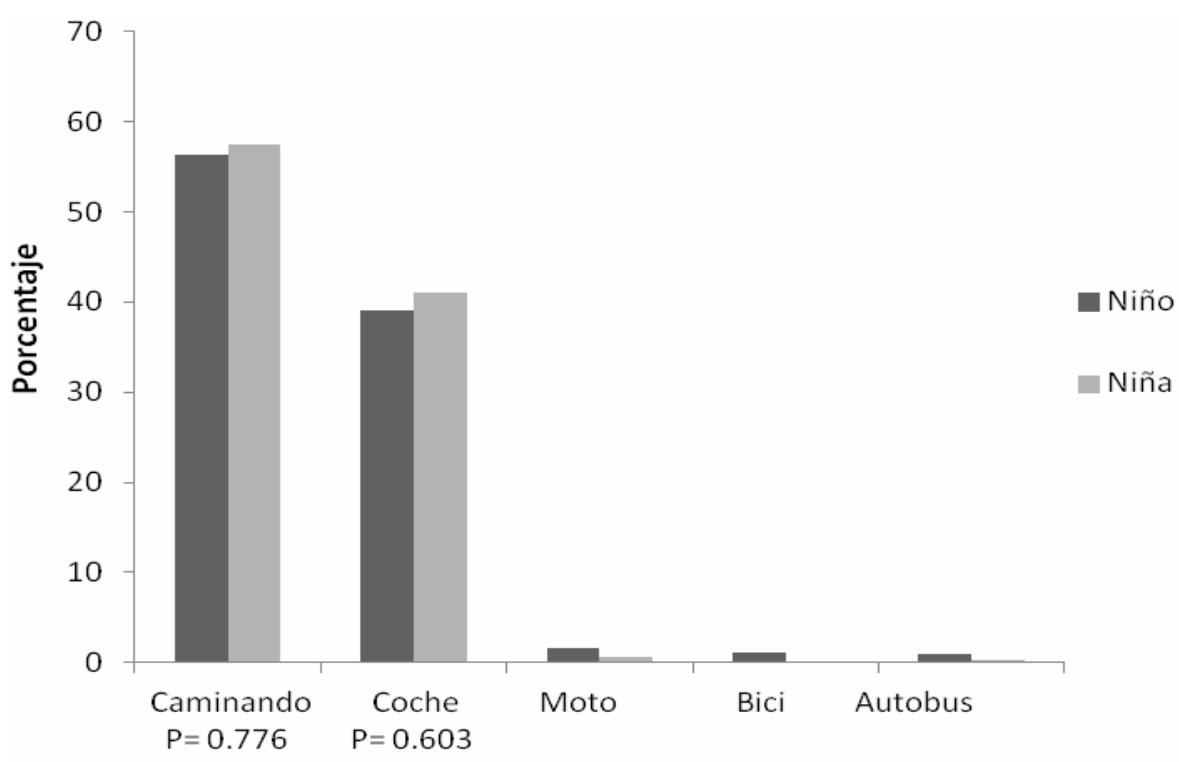

Figura 2. Modo de desplazamiento por género 


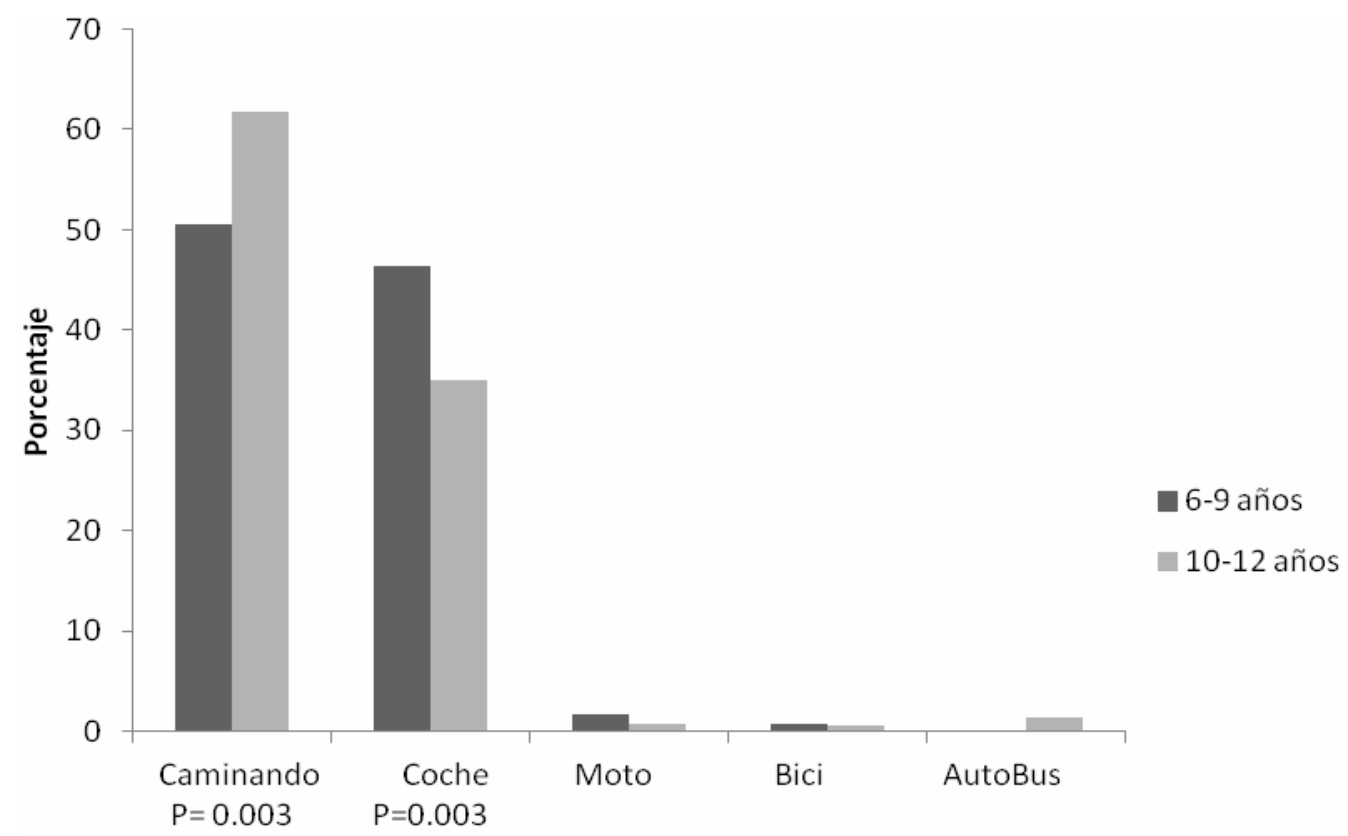

FIgura 3. Modo de desplazamiento por edad 


\section{INFORME}

PROYECTO "CAMINOS ESCOLARES SEGUROS Y SALUDABLES" CEIP REYES CATÓLICOS de SANTA FÉ

\section{INTRODUCCIÓN}

\subsection{Marco del Proyecto}

\subsection{Objetivos}

\subsection{Diseño de cuestionario}

\subsection{Procedimiento}

\subsection{Análisis de cuestionarios}

Primeramente se introdujeron en una base de datos para su posterior análisis estadístico todos los datos en bruto extraídos de los cuestionarios, con la previa creación de las variables pertinentes. La variable principal creada es el modo de desplazamiento al colegio y partiendo de ésta se creó una variable dicotómica para analizar el medio de transporte empleado para dicho desplazamiento: activo vs pasivo.

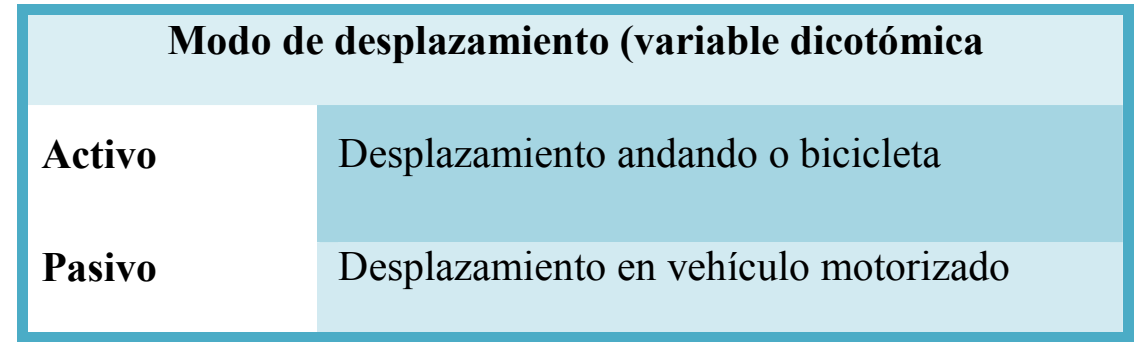

Seguidamente se procedió a:

1) Elección de las variables a estudiar

2) Codificación de los datos obtenidos en los cuestionarios

3) Diseño de la base de datos

4) Introducción de los datos en la misma

5) Análisis de resultados 
Es importante reseñar la disgregación por sexos que se realizó al inicio de la creación de la base de datos, aspecto que se consideró importante como hipótesis de partida, por si pudiera revelarse a la luz del análisis diferencias en los hábitos según género.

En cuanto a la extracción de datos para la realización de posteriores análisis, éstos se han segmentado por cursos.

Para el análisis de los datos se empleó el paquete estadístico SPSS Statistics versión 18.0 para Windows.

\section{CARACTERÍSTICAS SOCIODEMOGRÁFICAS}

Los participantes en el estudio fue el alumnado y sus respectivas familias (madres y padres) residentes en el municipio de Santa Fé. Las características de la muestra están expresadas en la tabla 1.

\begin{tabular}{ll}
\hline $\begin{array}{l}\text { NÚMERO DE } \\
\text { ENCUESTADOS }\end{array}$ & CEIP REYES CATÓLICOS \\
\hline Total alumnado & $\mathbf{1 5 1}$ participantes $(\mathbf{7 6}$ niños -75 niñas $)$ \\
\hline $3^{\circ}$ curso de Primaria & $\mathbf{2 4}(\mathbf{1 0}$ niños -14 niñas $)$ \\
\hline $4^{\text {o curso de Primaria }}$ & $\mathbf{3 4}(\mathbf{2 1}$ niños -13 niñas $)$ \\
\hline $5^{\circ}$ curso de Primaria & $\mathbf{3 4}(\mathbf{1 8}$ niños -16 niñas $)$ \\
$6^{\circ}$ curso de Primaria & $\mathbf{5 9}(27$ niños -32 niñas $)$ \\
Total familias & $\mathbf{1 3 0}$ participantes
\end{tabular}

Tabla 1. Características de la muestra 


\section{HÁBITOS DE DESPLAZAMIENTO}
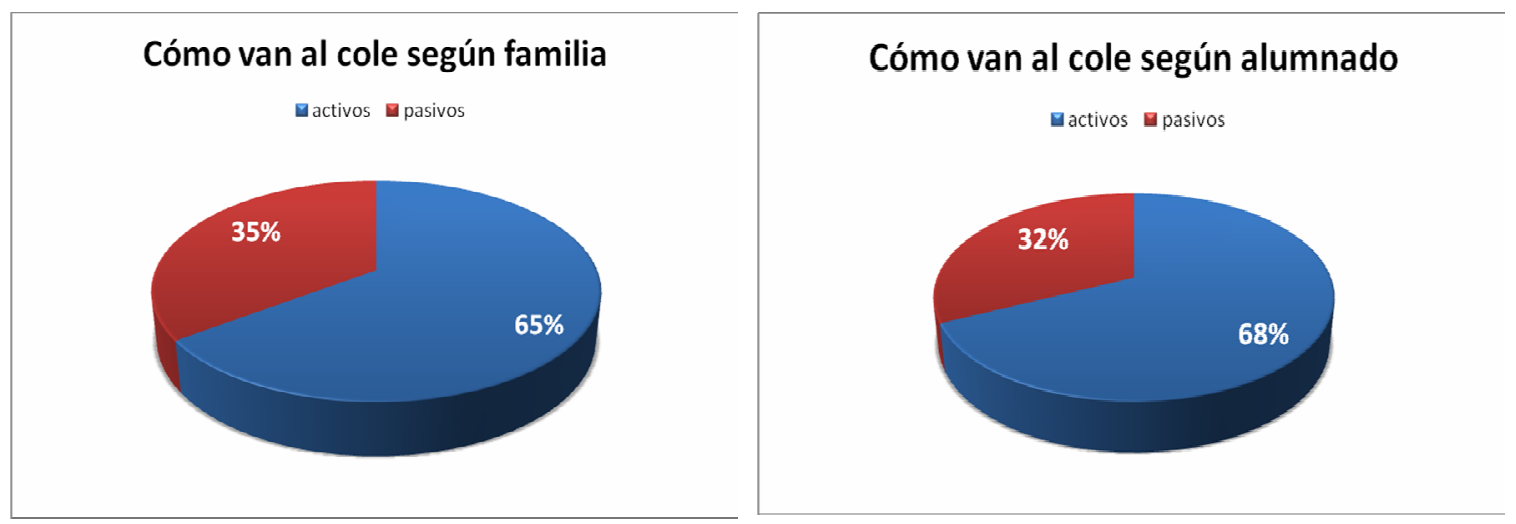

Figura 1. Modo de desplazamiento segmentado en activos y pasivos.

Un alto porcentaje del alumnado de Santa Fe $(>65 \%)$ va al colegio de manera activa, especialmente andando.

Algunos estudios demuestran que los chicos y chicas que se desplazan de forma activa al colegio tienen mejor capacidad cardiorrespiratoria que los que se desplazan de forma pasiva, entre otros beneficios.

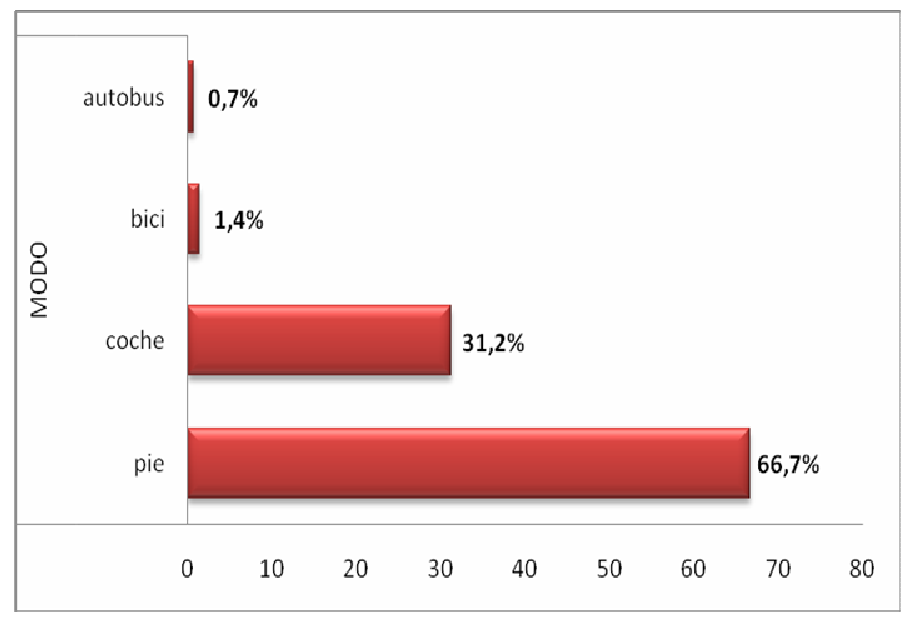

Figura 2. Medio de transporte con el que el alumnado va al colegio

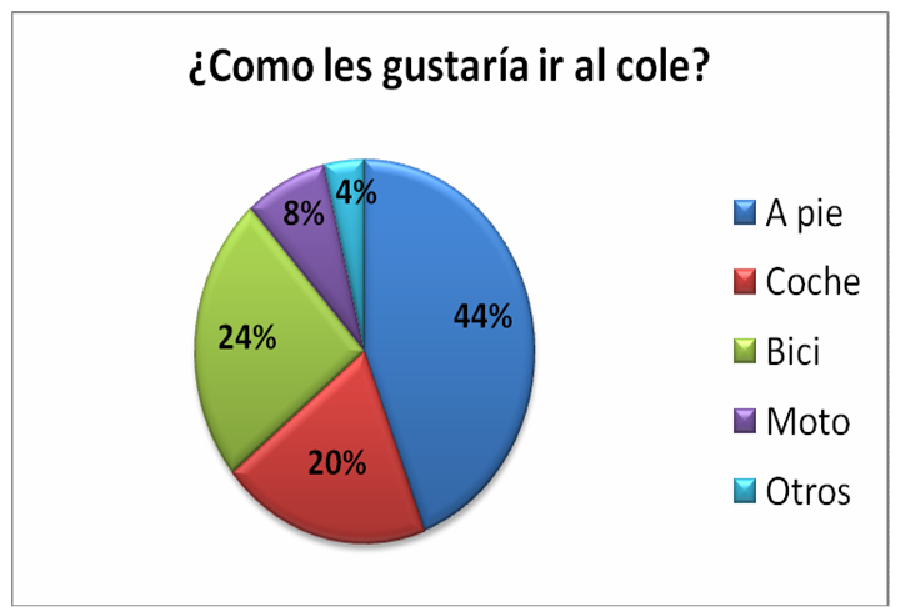

Figura 3. Medio de transporte que los niños y niñas prefieren para ir al colegio 
UN HAEIIO TAN SENGILLO GOMO ES IR ANDANDO AL GOLEGIO REPBRGUIE DE MANERA MUY PCEITIVA EN LA SALUD DE QUIEN LO PRAGTIGA, MEJORANDO LA GAPAGIDAD GARDIORESPIRATORIAY DIEMINUYANDO LOS NIVELIES DE ESTRESS Y ANEIEDAD

La figura 3 muestra las preferencias del alumnado de Santa Fé en el modo de transporte al colegio, destacando por encima del resto las formas activas (andando y en bicicleta). Y las razones de esta elección por parte de los niños y niñas se pueden apreciar en la figura 4, en la que se aprecia un alto porcentaje (casi el 30\%) de la razón "entretenimiento", que asociada a que casi el 70\% se desplaza a pie, y como se muestra más adelante, a que les gustaría realizar este trayecto acompañados de sus amigos/as, nos dan la respuesta a que andando y en sus propias bicicletas sean las opciones más deseadas en su manera de desplazarse al colegio.

Por tanto, no se debería utilizar el coche para llevar a los hijos al colegio si no es necesario, ya que con ello se les priva de una práctica tan saludable como es caminar y moverse en su entorno de forma más autónoma.

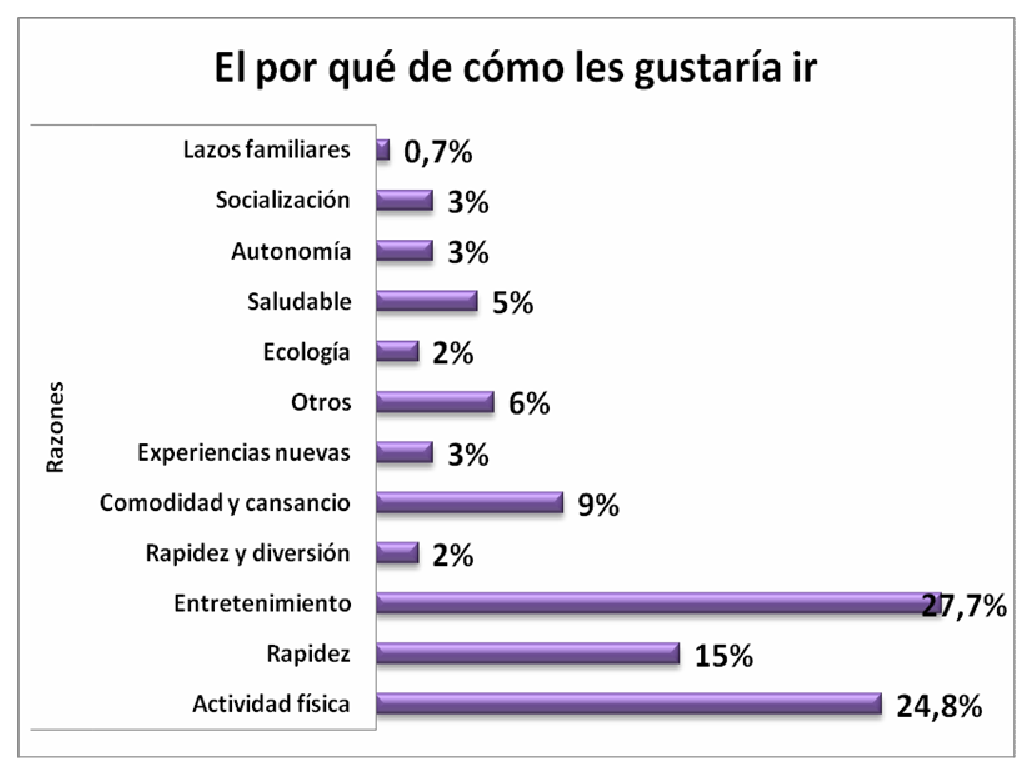

Figura 4. Razones por las que el alumnado prefiere otro modo de transporte al colegio 


\section{TIEMPOS Y DISTANCIAS}

En la siguiente figura se muestran las distancias desde el colegio al domicilio, según informaron las familias y el alumnado. Podemos observar que hay algunas diferencias, tal vez debido a que los niños y niñas a estas edades aún no son conscientes de las distancias y el saber apreciarlas, más aún si su desplazamiento se realiza en coche. Es por ello que se consideran más fiables los datos de los cuestionarios de las familias, al menos en cuanto a distancias y tiempo en el trayecto casa-cole.

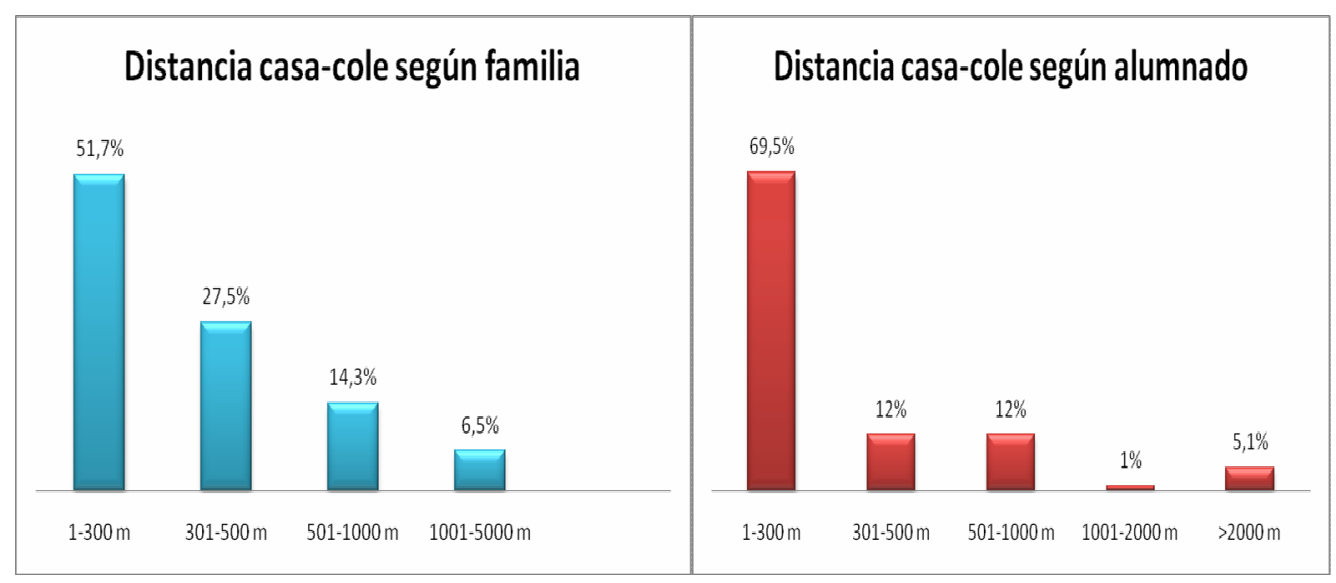

Figura 5. Distancia a la que viven del colegio el porcentaje total de alumnado.

Tomando como más fiables los resultados obtenidos de las familias, se puede confirmar que casi un $80 \%$ de las mismas viven o se desplazan menos de 500 metros para acceder al colegio, distancia totalmente realizable a pié. Si sumamos hasta $1 \mathrm{~km}$ de distancia, se obtiene que un $94 \%$ de las familias son potenciales para el transporte activo y susceptibles de poder ser intervenidas para mejorar dicho hábito.

De manera similar sucede con el tiempo empleado en recorrer esa distancia, en llegar al colegio. 


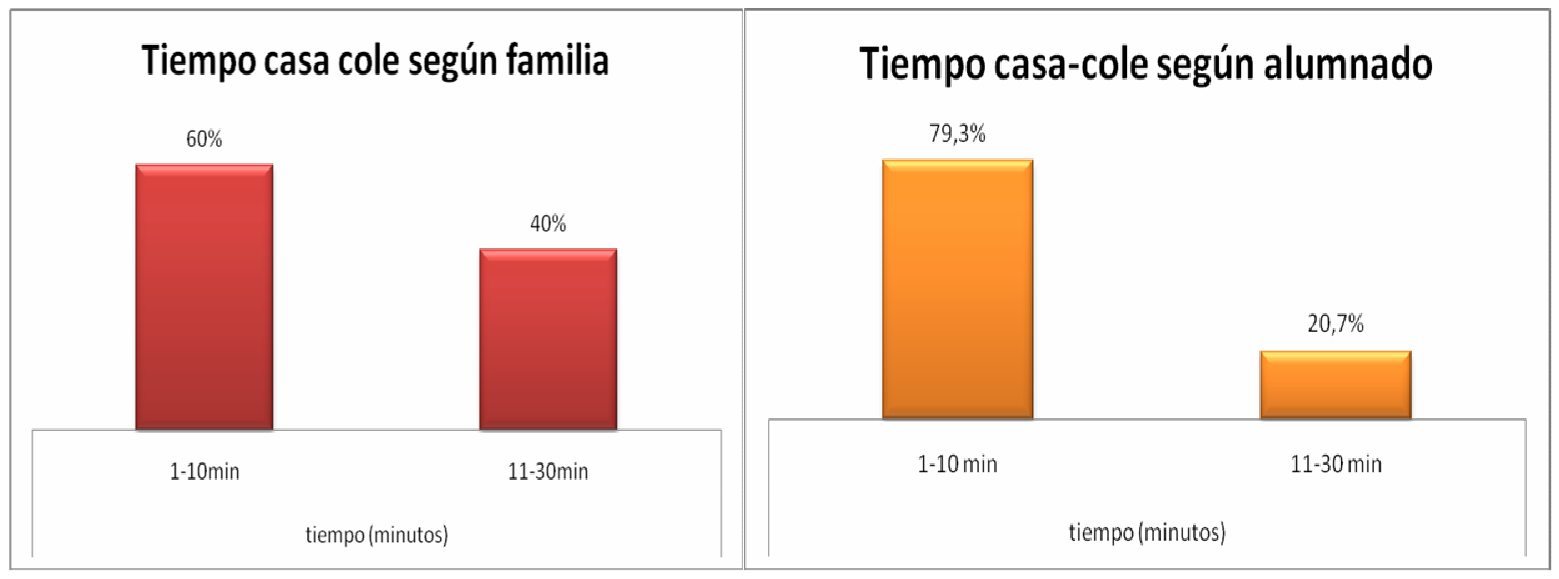

Figura 6. Tiempo en el trayecto desde el domicilio al colegio.

En la figura 6 se muestra el porcentaje total del alumnado del colegio estudiado, apreciándose que el tiempo empleado desde casa al colegio es de 10 minutos o menos para un elevado porcentaje (casi el 80\%) del alumnado, mientras que sólo el $60 \%$ de los padres aprecian que el tiempo empleado sea de menos de 10 minutos. El resto de padres (40\%), consideran que tardan más tiempo para realizar un trayecto de $1 \mathrm{~km}$. o menos, que como se comentaba anteriormente era la distancia a la que vivían del colegio el $94 \%$ de las familias.

Tras hacer una clasificación en activos (aquellos que se desplazan a pie o en bicicleta) y pasivos (aquellos que utilizan medios de transporte motorizados), teniendo en cuenta esta variable de distancia, los gráficos obtenidos muestran que para distancias inferiores a 10 minutos, el medio de transporte predominante es el activo, tanto para familias como para alumnado. En principio, parece existir coherencia entre la distancia declarada y el tiempo, pues el 94\% declara vivir menos de $1 \mathrm{~km}$ de distancia, que es la distancia que permitiría llegar andando en menos de 10 minutos. Parece ser que la percepción de la distancia y del tiempo es coherente, si bien no podemos asegurar el tiempo exacto que los participantes tardan en recorrer $1 \mathrm{~km}$. 


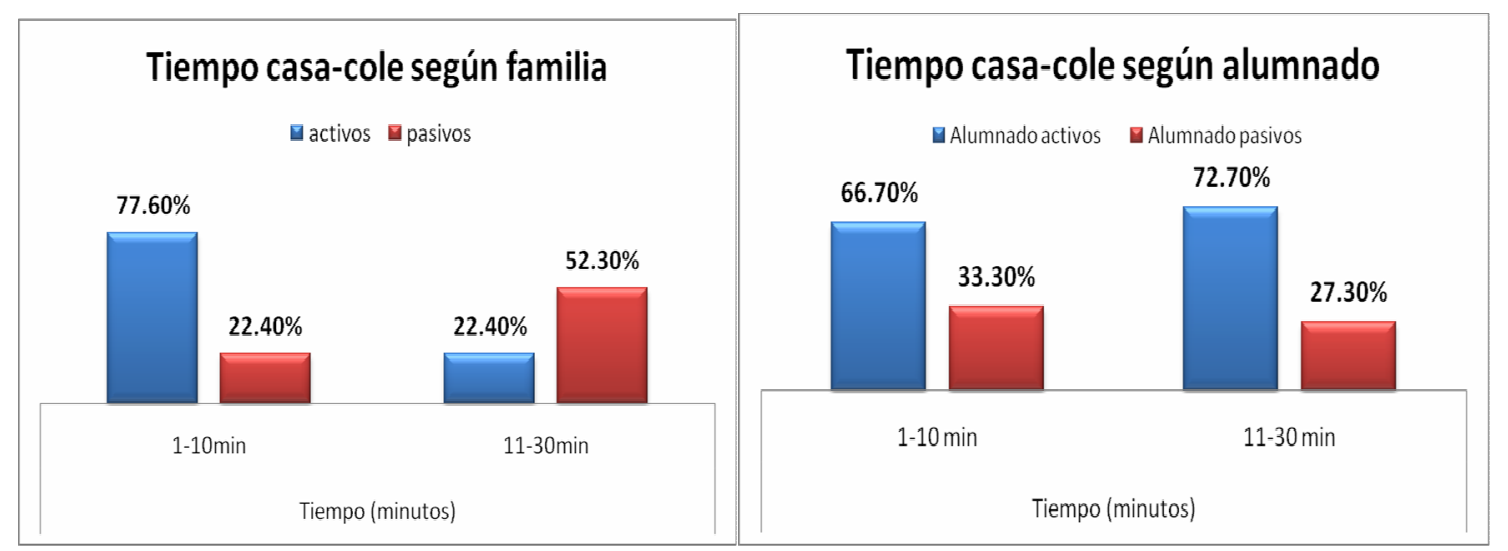

Figura 7. Tiempo en el trayecto desde el domicilio al colegio clasificado en activos y pasivos

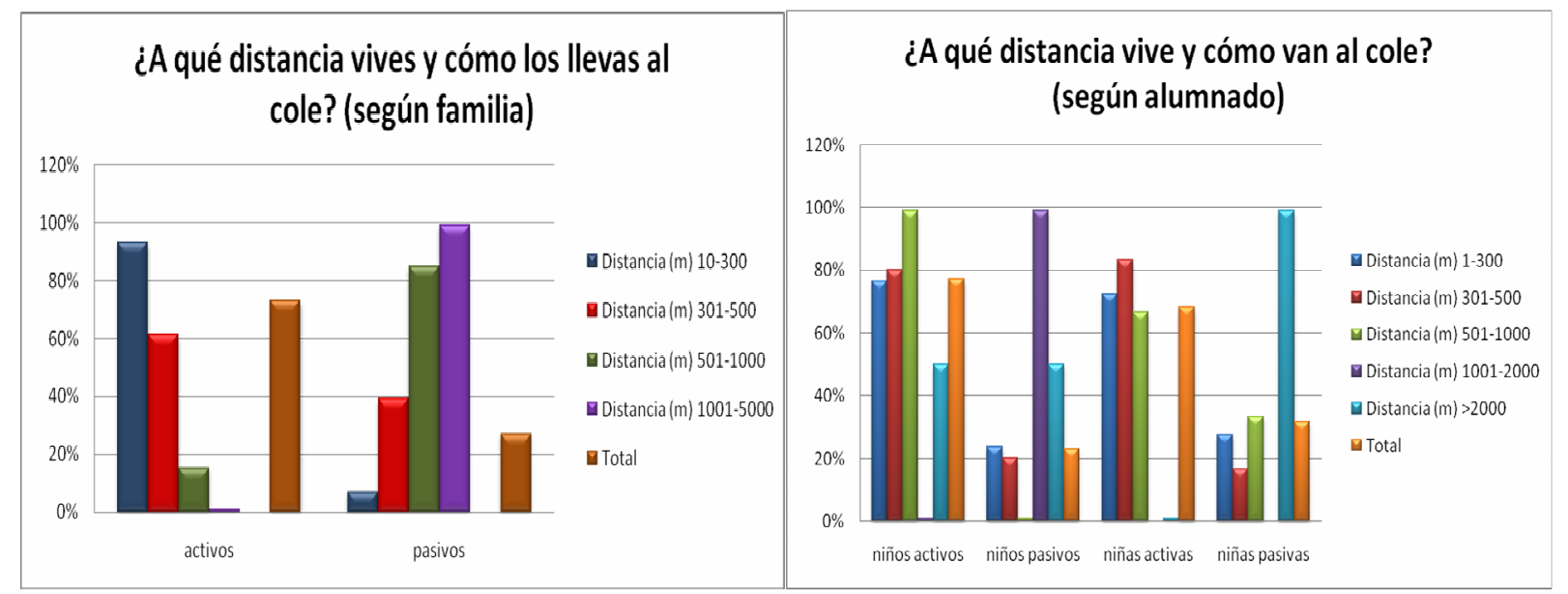

Figura 8. Modo de desplazamiento y distancias casa-cole.

Se aprecia sin embargo, un porcentaje relativamente alto de niño/as que van al colegio de manera pasiva (coche-moto-bus), aún viviendo entre 500 metros y 1 Kilómetro, y siempre atendiendo como más fiable la percepción de las familias, con porcentajes de casi el $85 \%$ de alumnado total que se desplaza de manera pasiva, si viven a más de $500 \mathrm{~m}$. 


\section{LA FORMA DE IR AL COLEGIO}

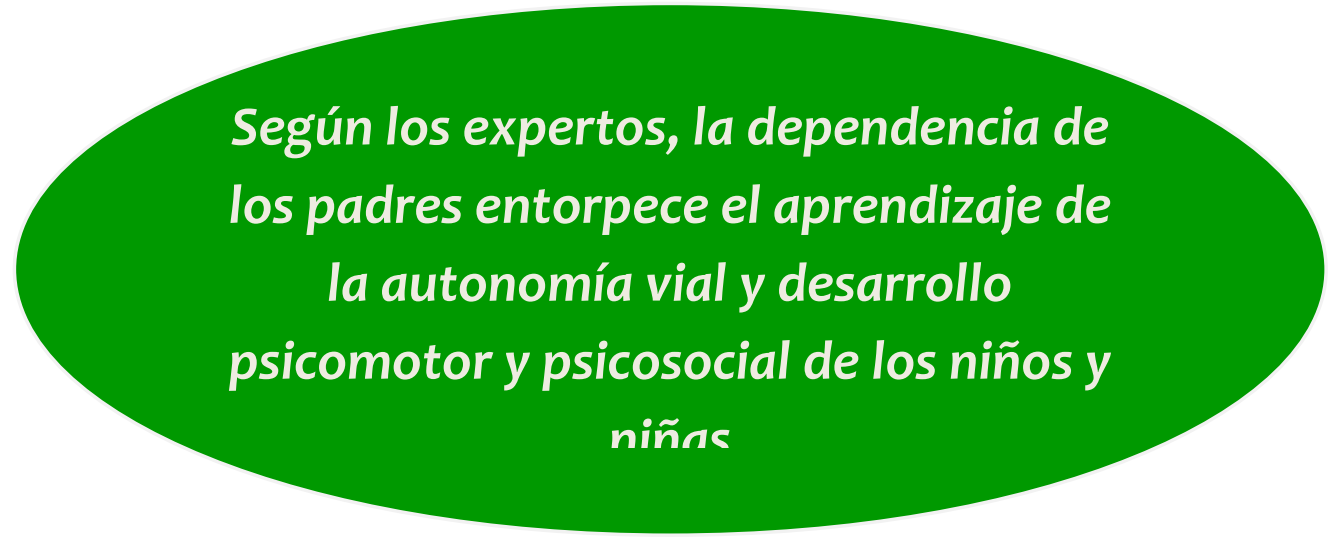

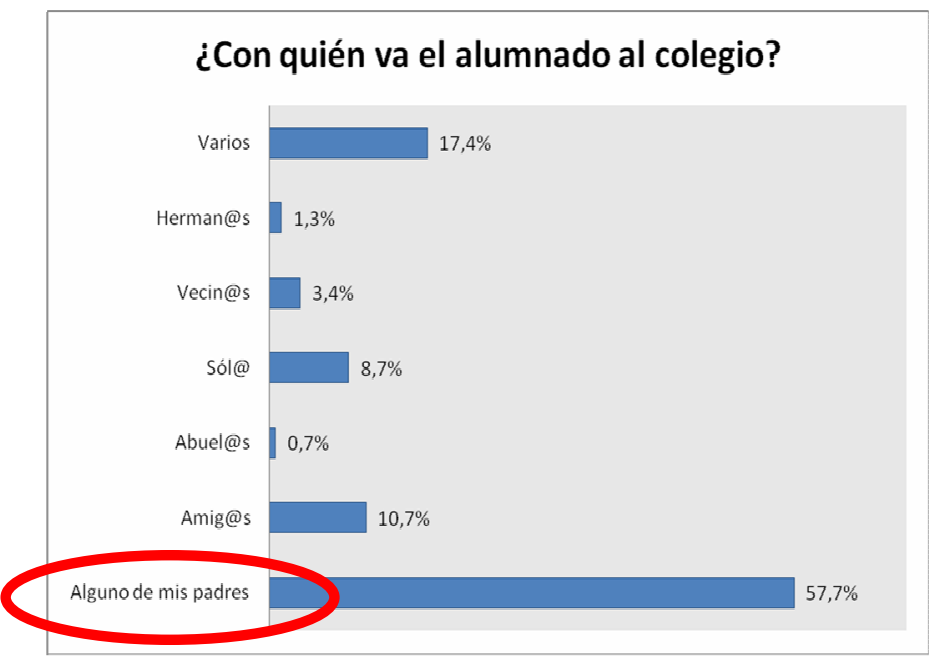

Figura 9. Personas que acompañan al alumnado al colegio.

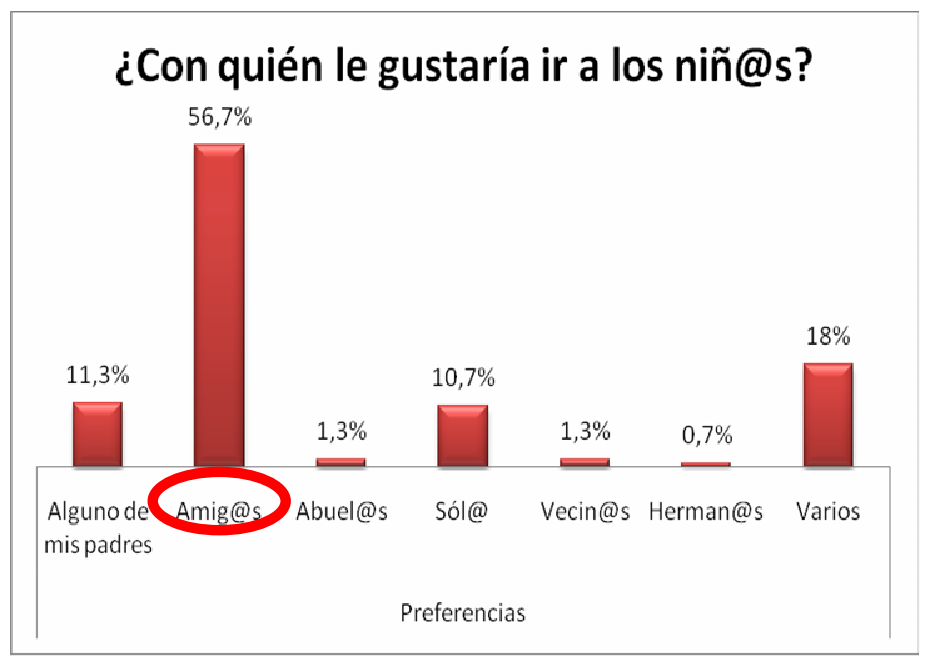

Figura 10. Preferencias del alumnado en el acompañamiento durante el trayecto casa-cole.

Más del 55\% del alumnado de Santa Fé realiza su trayecto al colegio acompañado por alguno de sus padres. Los datos de los cuestionarios de las familias nos muestran resultados muy similares. Sin embargo, como se ve en la figura 10, los niños y niñas prefieren ir acompañados de sus amigos y/o amigas, por un motivo principal: diversión y socialización.

\section{La edad media en la que los niños y niñas de Santa Fé comienzan a ir solos al colegio es a los 11 años}




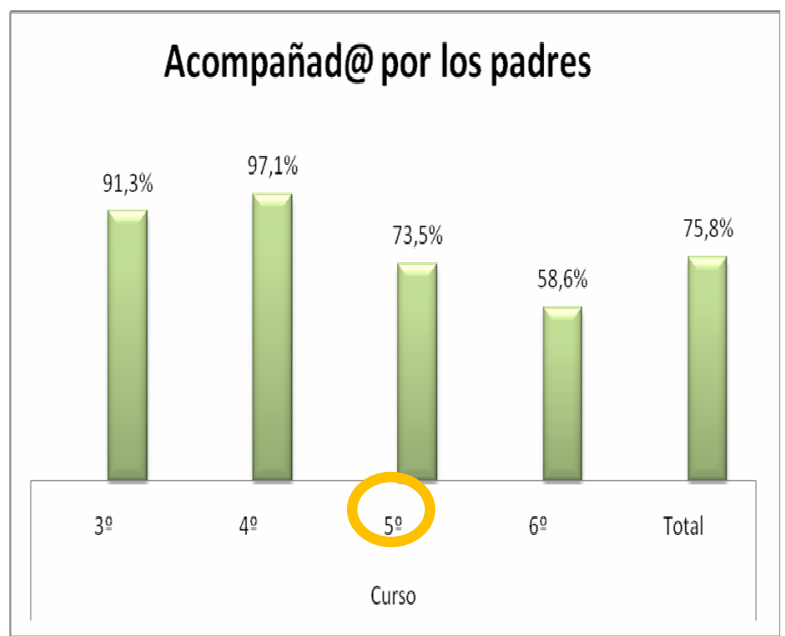

Figura 11. Acompañamiento de la familia

Según el gráfico, el curso en el que los niños y niñas empiezan a dejar de ir acompañados por alguno de sus padres es a partir de $5^{\circ}$ de Primaria. Pero cuando realmente empiezan a ser autónomos y algo más independientes en este aspecto es a partir de $6^{\circ}$ curso, aunque no sabemos qué sucede realmente a partir de este curso.

Sorprende que en $3^{\circ}$ haya más niños y niñas que acudan al colegio sin la compañía de sus padres que en $4^{\circ}$ curso. Estimamos que más de un $50 \%$ del alumnado acompañado y con 11 años de edad supera lo esperado por las capacidades que corresponden a esas edades.

Motivos de acompañara tu hij@

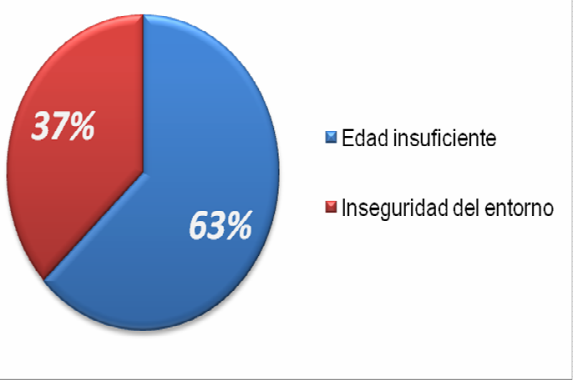

La mayoría de los padres que acompañan a sus hijos al colegio lo hacen por razones de edad, al considerarlos pequeños (63\%); el resto se encuentran intranquilos por la inseguridad que consideran que presenta el entorno.

Figura 12. Principales motivos por el que la familia acompañan a sus hijos/as 


\section{LAS CARACTERÍSTICAS DEL TRAYECTO}

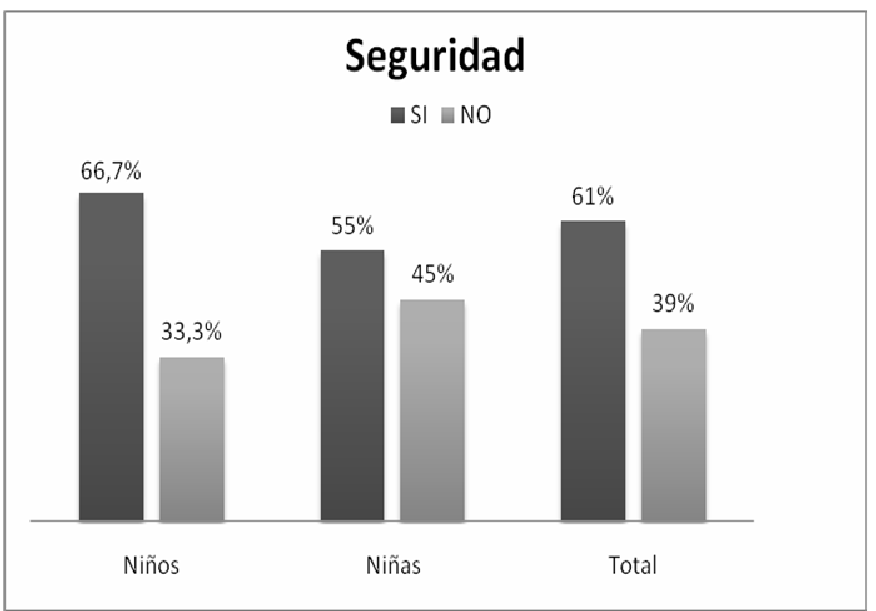

Figura 13. Percepción de seguridad del alumnado durante el trayecto.
En cuanto a la percepción de seguridad que tiene el alumnado sobre su trayecto de casa al colegio, el $61 \%$ comentan que encuentran una ruta segura.

Entre los motivos por los que los niños y niñas se sienten seguros, destacan el hecho de ir siempre acompañados. Por el contrario, los vehículos, el

tráfico y la gente desconocida o rara son los aspectos que más inseguridad producen, en el caso de que fueran solos al colegio.

Destaca la importancia de los temas relacionados con la seguridad ciudadana, tanto en la percepción de seguridad de las familias como en la de inseguridad percibida por los niños.

En la percepción de seguridad que tienen las familias sobre el entorno de sus hijos, la inseguridad (vial y ciudadana) es un factor más influyente que las deficiencias del entorno (urbanísticas...), tal y como se aprecia en la figura 14.

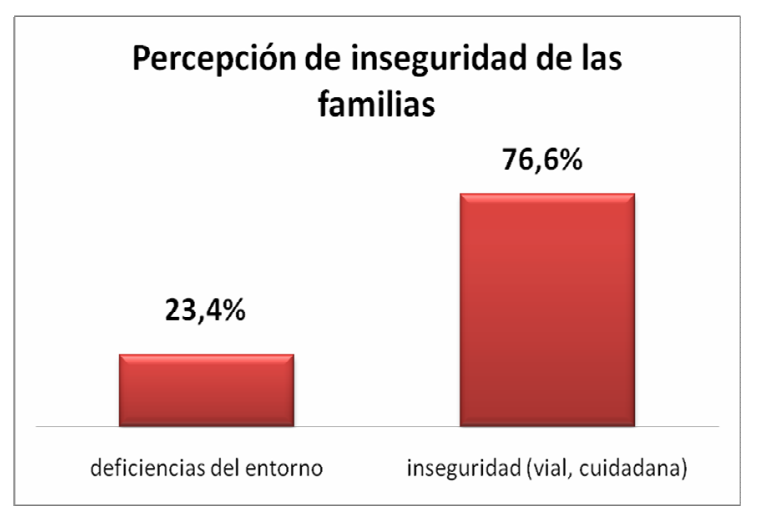

Figura 14. Percepción de inseguridad de las familias 


\section{PROPUESTAS FUTURAS Y DISPOSICIÓN ANTE LOS CAMBIOS}

En la siguiente figura se aprecian los posibles factores que les gusta a los alumnos cuando acuden al colegio de manera activa, ya sea andando o en bicicleta.

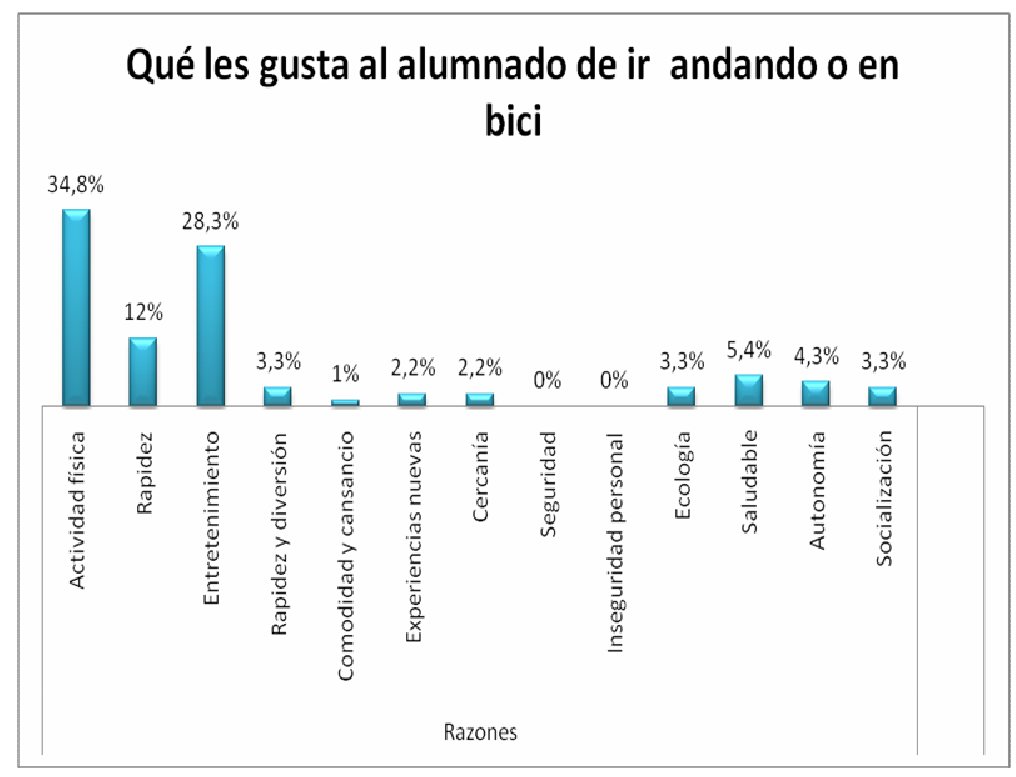

Figura 15. Factores que al alumnado le gusta de realizar su trayecto al colegio de manera activa.

Podemos apreciar como casi el 35\% del alumnado prefiere ir al colegio andando (el 33,7\%) o en bicicleta (el 1,1\%) por el claro hecho de la realización de actividad física, seguida por el entretenimiento (29\%) que la misma produce $(12 \%$ a pie y $16 \%$ en bici), los cuales se pueden asociar a los datos reflejados en la anterior figura 10 , en la que el alumnado prefiere ir al colegio con sus amigos y/o amigas.

A continuación se presentan las dificultades que encuentran tanto alumnado como familias para desplazarse al colegio de otra manera que no sea en coche ni moto. 


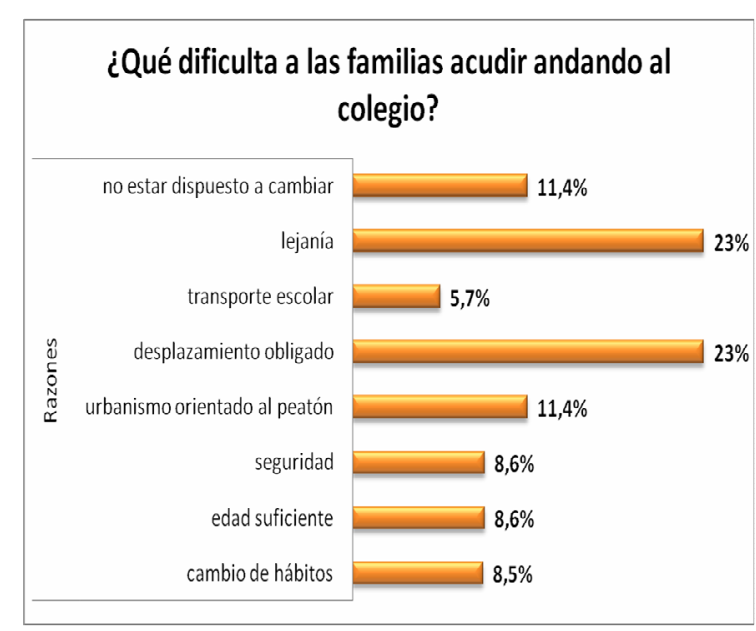

Figura 16. Razones que impiden que las familias dejen a sus hijos/as ir de manera activa al colegio ya sea solos o acompañados.

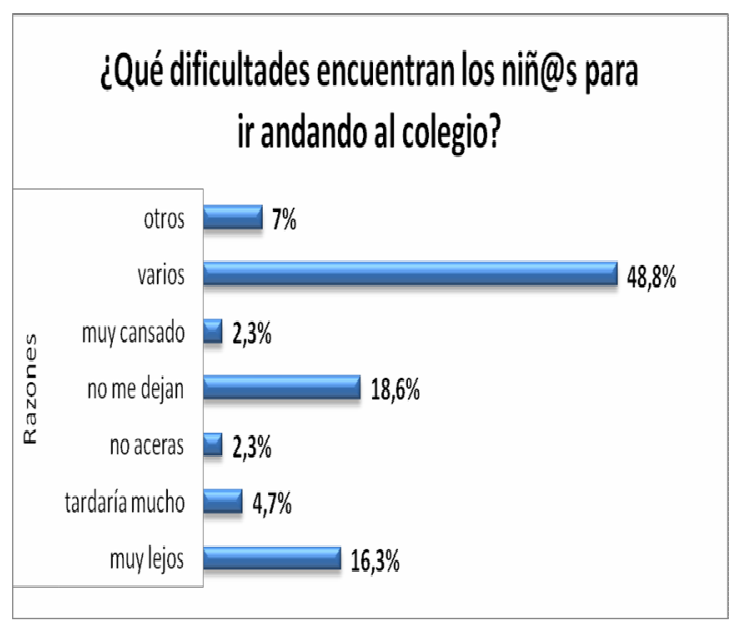

Figura 17. Dificultades que encuentra el alumnado para desplazarse de manera activa al colegio.

Dado que en los cuestionarios del alumnado no se indicaba la obligatoriedad de marcar una única respuesta, algunos marcaron varias de las respuestas, ya que consideraron que eran diversas las dificultades influyentes en la acción de ir al colegio andando; es por ello que la figura 17 nos muestra un alto porcentaje de "varios" (49\%).

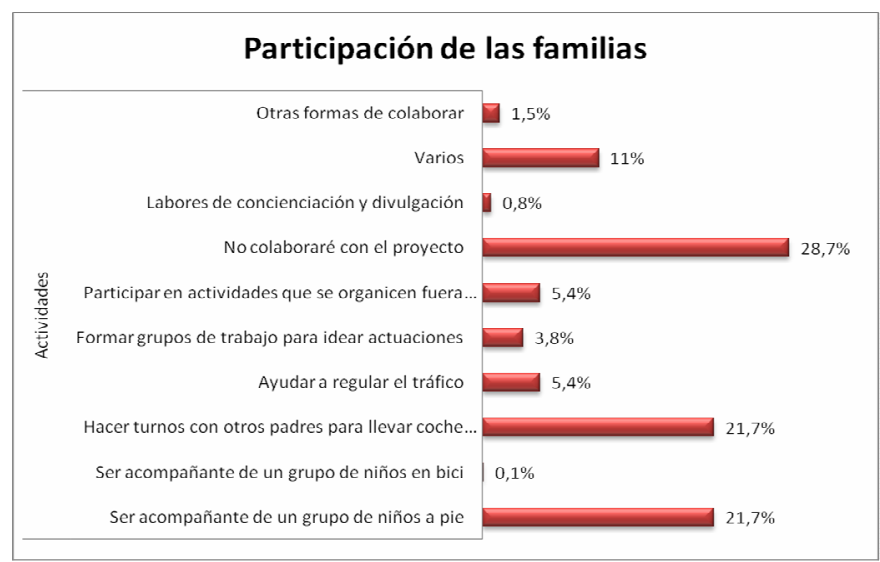

Figura 18. Actividades de colaboración de las familias

La figura 18 muestra en qué actividades estarían dispuestas a participar las familias para conseguir que sus hijo/a se desplacen de manera activa al colegio y evitando en mayor medida la aglomeración de vehículos a motor que se produce tanto a la entrada como a la salida del colegio.

Se observa como el "ser acompañante de un grupo de niños/as a pie" es la actividad en la que las familias estarían con mayor predisposición a realizar, cerca del 22\%. Mismo porcentaje que "el hacer turnos con otros padres para llevar a los hijos en coche". Por el 
contrario, existe un altísimo porcentaje (30\%) que no está dispuesto a intervenir ni a colaborar en las medidas.

\section{Posibles propuestas futuras}

Mantener el $65 \%$ del alumnado que se desplaza de forma activa al colegio

Modificar el hábito de desplazamiento de pasivo a activo del $84 \%$ del alumnado pasivos que vive a menos de un kilómetro.

Fomentar y facilitar el transporte en bicicleta, siendo este el interés actual del alumnado

Favorecer que el acompañamiento en distancias cortas de los activos al colegio, sea más de amigos que de familias.

Crear turnos de familias interesadas, que puedan acompañar al alumnado que vive a una distancia lejana del colegio.

Concienciar a las familias de la capacidad de movilidad independiente, que sus hijos tienen para desplazarse en edades tempranas.

Garantizar seguridad en familias y alumnado, creando rutas seguras. 
